\title{
A criterion for coarse iterability
}

\author{
Gunter Fuchs • Itay Neeman • Ralf Schindler
}

Received: 21 November 2006 / Accepted: 27 January 2010 / Published online: 16 March 2010

C The Author(s) 2010. This article is published with open access at Springerlink.com

\begin{abstract}
The main result of this paper is the following theorem: Let $M$ be a premouse with a top extender, $F$. Suppose that (a) $M$ is linearly coarsely iterable via hitting $F$ and its images, and (b) if $M^{*}$ is a linear iterate of $M$ as in (a), then $M^{*}$ is coarsely iterable with respect to iteration trees which do not use the top extender of $M^{*}$ and its images. Then $M$ is coarsely iterable.
\end{abstract}

Mathematics Subject Classification (2000) $\quad 03 E 45 \cdot 03 E 55$

Keywords Iterability $\cdot$ Iteration trees $\cdot$ Inner model theory $\cdot$ Large cardinals

During part of the research for this article, the first author was supported by DFG-grant Je 209/4-1, for which he wishes to express his gratitude. Also, during part of the research, he was affiliated to the Institute for Mathematical Logic and Foundations of Münster University, Germany.

G. Fuchs $(\bowtie)$

Department of Mathematics, College of Staten Island/Cuny, 2800 Victory Blvd, Staten Island,

NY 10314, USA

e-mail: Gunter.Fuchs@csi.cuny.edu

I. Neeman

Department of Mathematics, University of California at Los Angeles, Los Angeles,

CA 90095-1555, USA

R. Schindler

Institut für Mathematische Logik und Grundlagenforschung, Westfälische Wilhelms-Universität Münster, Einsteinstr. 62, 48149 Münster, Germany 


\section{Introduction}

In this article, we are going to prove a result concerning the iterability of premice. We assume the reader to be familiar with the theory developed in [2] or [1]. A good introduction to the area is the monograph [7]. Let's begin by fixing some terminology.

We shall deal with coarse normal iterations of premice. The term "premouse" here can be understood in the sense of [2] (or [4]), or in the sense of [1]. Both approaches use different kinds of indexing, but this difference shall not matter here.

Let $\mathcal{T}$ be a normal iteration tree in the sense of [1], or a normal $k$-maximal iteration tree in the sense of [2]. Such a tree comes with the sequences $\left\langle\kappa_{i}^{\mathcal{T}} \mid i+1<\operatorname{lh}(\mathcal{T})\right\rangle$, $\left\langle\lambda_{i}^{\mathcal{T}} \mid i+1<\operatorname{lh}(\mathcal{T})\right\rangle$ and $\left\langle v_{i}^{\mathcal{T}} \mid i+1<\operatorname{lh}(\mathcal{T})\right\rangle$ of the critical points of the extenders applied, their lengths, and their indices, respectively.

We write $\mathcal{T}(i+1)$ for the immediate $\mathcal{T}$-predecessor of $i+1$ in the tree-order $<\mathcal{T}$, which is denoted by $\mathcal{T}-\operatorname{pred}(i+1)$ in [2], if $i+1<\operatorname{lh}(\mathcal{T})$. Also, in case the $i$ th model of the iteration tree is active, we shall write $\hat{\kappa}_{i}^{\mathcal{T}}$ for the critical point of $E_{\text {top }}^{\mathcal{M}_{i}^{\mathcal{T}}}$, that is, of the top extender of that model, and $\hat{\lambda}_{i}^{\mathcal{T}}$ for its "length", according to the particular indexing scheme used. So $\hat{\lambda}_{i}^{\mathcal{T}}$ is the image of the critical point of the extender under the associated embedding, if Jensen indexing is used, and it is the strict supremum of the generators of the extender and the ordinals less than the successor of its critical point, as computed in the premouse, if Mitchell-Steel indexing is used. $\hat{v}_{i}^{\mathcal{T}}$ stands for the index of the top extender, that is, for the height of $\mathcal{M}_{i}^{\mathcal{T}}$.

Finally, we let $\widehat{\mathcal{T}}(i+1)$ be the minimal $\xi$ s.t. $\xi=i$, or else $\xi<i$ and $\hat{\kappa}_{i}<\lambda_{\xi}^{\mathcal{T}}$ (for $i<\operatorname{lh}(\mathcal{T})$ ). So it is the index to which the top extender of the $i$ th model in the iteration would have to be applied, according to the rules for normal iterations. Hence for every normal $\mathcal{T}, \widehat{\mathcal{T}}(i+1)=\mathcal{T}(i+1)$ for every $i<\operatorname{lh}(\mathcal{T})$.

Frequently, when it is clear which iteration tree we are referring to, the superscript $\mathcal{T}$ will be omitted.

A coarse ultrapower (often called $\Sigma_{0}$ ultrapower) is an ultrapower which is formed by using only functions which are elements of the structure one wants to take the ultrapower of, whereas a fine ultrapower is an ultrapower which is formed by using functions in the appropriate definability level over that structure. By a coarse normal iteration, we mean an iteration in which we use coarse ultrapowers for forming $\mathcal{M}_{i+1}^{\mathcal{T}}=\operatorname{ult}\left(\mathcal{M}_{i}^{*}, E_{i}^{\mathcal{T}}\right)$ (where $\mathcal{M}_{i}^{*}$ is the model to which $E_{i}^{\mathcal{T}}$ has to be applied according to the rules for forming normal iterations) if there are no drops in $[0, i)_{\mathcal{T}}$ (no matter if $E_{i}^{\mathcal{T}}$ is the top extender of $\mathcal{M}_{i}^{\mathcal{T}}$ or not). Otherwise, the ultrapowers are formed as fine as possible, depending on where the critical point of $E_{i}^{\mathcal{T}}$ lies in the sequence of the projecta of $\mathcal{M}_{i}^{*}$.

Let us now state more precisely what we are going to prove.

Definition 1.1 Let $M$ be an active premouse. We let $M^{\text {passive }}$ be the premouse obtained from $M$ by omitting the top extender.

The coarse top iteration of $M$ of length $\theta$ is the linear normal coarse iteration of $M$ of length $\theta$, in which always the top extender is applied (if it exists, that is, if the models in the iteration all are well founded). So it is the 0 -maximal iteration tree $\mathcal{T}$ on $M$ of length $\theta$ such that $v_{i}^{\mathcal{T}}=\hat{v}_{i}^{\mathcal{T}}$, for all $i$ with $i+1<\theta$. 
We say that $M$ is coarsely $\alpha$-iterable by its top extender iff the coarse top iteration of $M$ of length $\alpha$ exists. $M$ is coarsely $\alpha$-iterable iff there is an iteration strategy $\Sigma$ for $M$ with respect to trees on $M$ which are coarse and normal and have length less than $\alpha$.

$M$ is coarsely iterable by its top extender iff it is coarsely $\alpha$-iterable by its top extender, for every $\alpha . M$ is coarsely normally iterable iff there is an iteration strategy $\Sigma$ for $M$ with respect to trees on $M$ which are coarse and normal and of arbitrary (set sized) length.

$N$ is a coarse top iterate of $M$ if there is a coarse top iteration of $M$ with last model $N$. $N$ has stage $\alpha$ if this top iteration has length $\alpha+1$.

Finally, we say that $M$ is separately $\alpha$-iterable if $M$ is coarsely $\alpha$-iterable by its top extender, and if for every coarse top iterate $N$ of $M$ with stage $<\alpha, N^{\text {passive }}$ is coarsely normally $\alpha$-iterable.

Our main result, Theorem 4.1, is:

Main Theorem Let $M$ be an active premouse. If $M$ is separately $\alpha+1$-iterable, then $M$ is coarsely normally $\alpha+1$-iterable.

The following is Corollary 4.2. The hypothesis on the definability of $\left\langle\Sigma_{\alpha} \mid \alpha<\infty\right\rangle$ is somewhat awkward, but it is necessary to allow piecing together the individual strategies to one strategy which works for all set sized (coarse and normal) trees.

Corollary If $M$ is separately $\alpha$-iterable, for every $\alpha$, and if the sequence of the corresponding iteration strategies $\left\langle\Sigma_{\alpha} \mid \alpha<\infty\right\rangle$ is definable, where $\Sigma_{\alpha}$ is an $\alpha+1$-iteration strategy of the coarse top iterate of stage $\alpha$ of $M$, then $M$ is coarsely normally iterable.

The authors would like to express their gratitude to the referee, whose very helpful comments improved the paper considerably.

\section{An application}

To give an example application of these results, let's assume that $x^{\sharp}$ exists, and that there is no inner model with a Woodin cardinal. This application might play a role in a future analysis of the strength of " $u_{2}=\aleph_{2}$."

The assumption that $x^{\sharp}$ exists gives us "enough of a measurable cardinal" in $L[x]$ to carry out the construction of $K$ there - we apply the theory developed in [3]; there, one working assumption is that there is no inner model with a Woodin cardinal, and that $\Omega$ is measurable. Then $K^{c}$ and $K$ are built, giving universal "weasels" of height $\Omega$. But in order for the construction to work, it is not necessary to have the normal ultrafilter on $\Omega$ be a set, instead, it suffices to have a "V-ultrafilter". So using the normal ultrafilters on the $x$-indiscernibles, we can build $K$ in $L[x]$ up to any of those indiscernibles, and those mice stack up to what we refer to as $K=K^{L[x]}$. See $[3$, p. 58] for a detailed discussion of this.

Let $x^{\sharp}=\left\langle J_{\nu}[x], F\right\rangle$. We would like to "add $F$ as a top extender to $K \| v$ ", but in order for this to work, we have to be a little more careful, because we want the structure to be a premouse. For notational convenience, we shall here use $\lambda$-indexing, as in [1], as well as the functional representation of extenders. So $F$ is a function from 
$\mathcal{P}(\kappa) \cap J_{v}[x]$ to $\mathcal{P}(\lambda)$, where $\kappa$ is the critical point of $F$, i.e., the least $x$-indiscernible, and $\lambda=F(\kappa)$. Let $\tau=\left(\kappa^{+}\right)^{K}$ (actually, it follows from [3, Thm. 1.4.] that $\left.\tau=\left(\kappa^{+}\right)^{L[x]}\right)$.

For $\kappa<\alpha \leq \lambda$, let $F \mid \alpha: \operatorname{dom}(F) \longrightarrow \mathcal{P}(\alpha)$ be the extender defined by stipulating that $(F \mid \alpha)(x)=F(x) \cap \alpha$. Further, let $\tilde{K}_{\alpha}=\operatorname{ult}(K \| \tau, F \mid \alpha)$, and let $\pi_{\alpha}$ be the ultrapower embedding. Set $K^{\prime \alpha}=\left\langle\tilde{K}_{\alpha}, \pi_{\alpha}\left\lceil\left(\mathcal{P}(\kappa) \cap \tilde{K}_{\alpha}\right)\right\rangle\right.$. Then $K^{\prime \alpha}$ is almost a premouse; only the initial segment condition might fail. But there is a maximal $\alpha_{0} \in(\kappa, \lambda]$ such that $K^{\prime \alpha_{0}}$ does satisfy the initial segment condition. Let $K^{\prime}=K^{\prime \alpha_{0}}$. We show:

Lemma 2.1 $K^{\prime}$ is a coarsely normally iterable premouse.

Proof By our main theorem, showing that every top iterate of $K^{\prime}$ is coarsely normally iterable with respect to iterations not using the top extender, and that the sequence of those coarse normal iteration strategies is definable, is more than enough for this.

Let $K_{\alpha}^{\prime}$ be the coarse top iterate of $K^{\prime}$ of stage $\alpha$, with embeddings $\bar{\pi}_{i, j}$, and let $\left\langle N_{\gamma} \mid \gamma<\infty\right\rangle$ be the top iteration of $x^{\sharp}$, with embeddings $\pi_{i, j}$. Now we have an elementary embedding $\sigma_{0}: K^{\prime \text { passive }} \longrightarrow K \| v$, defined as follows: Letting $\bar{\pi}$ : $K \| \tau \longrightarrow_{F \mid \alpha_{0}} K^{\prime \text { passive }}$ and $\pi: J_{\tau}[x] \longrightarrow_{F} J_{\nu}[x]$, we can set $\sigma_{0}(\bar{\pi}(f)(\vec{\gamma}))=$ $\pi(f)(\vec{\gamma})$ (where $f: \kappa \longrightarrow K\|\tau, f \in K\| \tau$ and $\vec{\gamma}<\alpha_{0}$ ). This works because $K \| \tau$ is definable in $J_{\tau}[x]$, by the local definability of $K$. But then we have $\sigma_{0}$ : $\left\langle\left|K^{\prime}\right|, E_{\text {top }}^{K^{\prime}}\right\rangle \longrightarrow_{0}\left\langle J_{v}[x], F\right\rangle$ meaning that $\sigma_{0}:\left\langle\left|K^{\prime}\right|, \in\right\rangle \longrightarrow_{\Sigma_{0}}\left\langle\left|J_{v}[x]\right|, \in\right\rangle$, and that for $x \in \mathcal{P}(\kappa)$ and $\gamma<\alpha_{0}, \gamma \in E_{\text {top }}^{K^{\prime}}(x)$ iff $\sigma_{0}(\gamma) \in F\left(\sigma_{0}(x)\right)$. Actually, since $\sigma_{0}\left\lceil\alpha_{0}=\mathrm{id}\right.$, the latter is equivalent to $\gamma \in F(x)$, but this simple reduction won't work in later iterates anymore.

So, an obvious copying construction inductively gives us embeddings $\sigma_{i}: K_{i}^{\text {passive }}$ $\longrightarrow K^{N_{i}}=K \mid \operatorname{ht}\left(N_{i}\right)$, so that $\sigma_{i}:\left\langle\left|K_{i}^{\prime}\right|, E_{\mathrm{top}}^{K^{\prime}}\right\rangle \longrightarrow_{0}\left\langle\left|N_{i}\right|, E_{\mathrm{top}}^{N_{i}}\right\rangle$, and so that the embeddings commute: $\sigma_{j} \bar{\pi}_{i, j}=\pi_{i, j} \sigma_{i}$. The definition in the successor case is $\sigma_{i+1}\left(\bar{\pi}_{i, i+1}(f)(\vec{\gamma})\right)=\pi_{i, i+1}\left(\sigma_{i}(f)\right)\left(\sigma_{i}(\vec{\gamma})\right)$, and the definition in the limit case is just as obvious (and works because the embeddings commute). We will have that for $i \leq j, \sigma_{i}\left\lceil\operatorname{lh}\left(E_{\mathrm{top}}^{K_{i}^{\prime}}\right)=\sigma_{j}\left\lceil\operatorname{lh}\left(E_{\mathrm{top}}^{K_{i}^{\prime}}\right)\right.\right.$.

The last point is that since we assumed that there is no inner model with a Woodin cardinal, it follows that $K$ is $(\omega, \infty)$-iterable, not only in $L[x]$, where it was built, but also in V. To this end, it suffices to show that arbitrarily long proper initial segments of $K$ are iterable.

So fix such a segment of $K$, say $K \| \beta$. We may pick $\beta$ so that $\beta$ is a $K$-cardinal; in particular, $K \| \beta$ sees no Woodin cardinal. Moreover, $K \| \beta$ is 1 -small. The argument to follows is part of the folklore and in fact shows that in the absence of an inner model with a Woodin cardinal the iterability of premice with no (definable) Woodin cardinal is absolute between transitive models which contain $\omega_{1}^{\mathrm{V}}$.

We claim that $K \| \beta$ is normally iterable by the following potential strategy, call it $\Sigma$. If $\mathcal{T}$ is a normal tree on $K \| \beta$ of limit length $\lambda$, then we let $\Sigma(\mathcal{T})$ be the (unique) cofinal branch $b$ through $\mathcal{T}$, if it exists, such that there is some $\gamma \geq \delta(\mathcal{T}), \gamma$ less than or equal to the ordinal height of $M_{b}^{\mathcal{T}}$, such that $M_{b}^{\mathcal{T}} \| \gamma$ is a $Q$-structure for the 
common part model $M(\mathcal{T})$ of $\mathcal{T}{ }^{1}$ (If this $Q$-structure exists, then it is in fact of the form $J_{\gamma}[M(\mathcal{T})]$.) If there is no such branch $b$, then $\Sigma(\mathcal{T})$ is undefined.

Let $U \in L[x]$ be a tree of height $\omega$ of attempts to find

(a) a countable transitive model $\bar{K}$ together with an elementary embedding $\pi: \bar{K} \rightarrow$ $K \| \beta$ and

(b) a transitive model $H$ of $\mathrm{ZFC}^{-}$(where we may witness the transitivity by embedding the ordinals of $H$ into $\omega_{1}^{\mathrm{V}}$ ) such that

(c) $H \models$ "there is no inner model with a Woodin cardinal, and there is a countable putative normal iteration tree $\mathcal{T}$ on $\bar{K}$ which either has a last ill-founded model or else there is no maximal branch $b$ through $\mathcal{T}$ such that if $\lambda=\sup (b) \leq \operatorname{lh}(\mathcal{T})$ and $\gamma \geq \delta(\mathcal{T} \uparrow \lambda)$ is least such that $\delta(\mathcal{T} \uparrow \lambda)$ is not definably Woodin over $J_{\gamma}[M(\mathcal{T} \uparrow$ $\lambda)]$, then $J_{\gamma}\left[M(\mathcal{T}\lceil\lambda)]\right.$ is (isomorphic to) and initial segement of $M_{b}^{\mathcal{T}}$."

Let's assume, towards a contradiction, that $K \| \beta$ is not iterable by $\Sigma$. By forcing over the transitive collapse of an appropriate Skolem hull of a rank initial segment of V, we may then argue that $U$ is ill-founded in $\mathrm{V}$. This implies that $U$ is also ill-founded in $L[x]$, so that $K \| \beta$ would not be iterable by $\Sigma$ in $L[x]$. But this is a contradiction!

This argument uses that the statament in $(c)$ above about the nonexistence of $b$ can be written in a $\Pi_{1}^{1}$-fashion in the appropriate parameters and is hence absolute between $\mathrm{V}, L[x]$, and the countable transitive model $H$.

So we get an iteration strategy for $K_{\gamma}^{\prime}$ passive, which is the "pullback" of the strategy for $K$, and which consequently only depends on the canonical embedding from $K_{\gamma}^{\prime}$ passive into the corresponding segment of $K$. Since the sequence of embeddings is clearly definable from $x^{\sharp}$, so is the sequence of iteration strategies.

Now Corollary 4.2 to our Main Theorem tells us that $K^{\prime}$ is coarsely normally iterable, as wished.

Another application was pointed out to us by the referee. Let $\lambda$ be an infinite cardinal, and let $M$ be an active premouse of size bigger than $\lambda$. Suppose that the top extender of $M$ is $\lambda$-complete (cf. [6, Definition 8.11]). Suppose also that if $\pi: N \rightarrow M^{\text {passive }}$ is elementary, where $N$ is transitive and of size $\lambda$, then $N$ is coarsely normally $\lambda^{+}+1$-iterable. Then every transitive $N^{\prime}$ of size $\lambda$ such that there is an elementary $\pi: N^{\prime} \rightarrow M$ is coarsely normally $\lambda^{+}+1$-iterable. This is shown by observing that if $N^{\prime}$ is transitive and of size $\lambda$ such that there is some elementary $\pi: N^{\prime} \rightarrow M$, then $N^{\prime}$ is coarsely $\lambda^{+}+1$-iterable by its top extender provided that this top extender is $\lambda$-complete.

\section{Some machinery}

We collect in this section some key observations which are needed in order to carry out some kind of a copying construction in the proof of the main result.

Let $\mathcal{T}$ be a normal iteration tree.

Lemma 3.1 Let $i<\operatorname{lh}(\mathcal{T})$ be such that there is no truncation in $[0, i]_{\mathcal{T}}$, and let $\xi=\widehat{\mathcal{T}}(i+1)$. Then:

\footnotetext{
1 As a reference for both terminology and results about unique cofinal branches and $Q$-structures, we refer the reader to $[4,6.9-6.14]$.
} 
(a) $\xi \leq \mathcal{T} i$ and $\hat{\kappa}_{\xi}=\hat{\kappa}_{i}$.

(b) There is no $h+1 \in(\xi, i]_{\mathcal{T}}$ such that $v_{h}=\hat{v}_{h}$.

Proof of (a) If $\xi=i$, then we are done. So let $\xi<i$, and assume, towards a contradiction, that $\xi \nless_{\mathcal{T}} i$. Let $h<i$ be minimal s.t. $\bar{h}:=\mathcal{T}(h+1)<\xi<h+1 \leq \mathcal{T} i$. So

(1) $\kappa_{h}<\lambda_{\bar{h}} \leq \hat{\kappa}_{i}$.

(2) $\hat{\kappa}_{\bar{h}}<\kappa_{h}$.

Proof Otherwise, $\hat{\kappa}_{h+1}=i_{\bar{h}, h+1}^{\mathcal{T}}\left(\hat{\kappa}_{\bar{h}}\right) \geq \lambda_{h}$, so, since $h+1 \leq \mathcal{T} i, \hat{\kappa}_{i} \geq \lambda_{h}$. But $\widehat{\mathcal{T}}(i+1)=\xi \leq h$, so $\hat{\kappa}_{i}<\lambda_{h}$, a contradiction.

So since $\hat{\kappa}_{h+1}=i i_{\bar{h}, h+1}^{\mathcal{T}}\left(\hat{\kappa}_{\bar{h}}\right)$, it follows that

(3) $\hat{\kappa}_{h+1}=\hat{\kappa}_{\bar{h}}$.

$\operatorname{But} \operatorname{crit}\left(i_{h+1, i}^{\mathcal{T}}\right) \geq \lambda_{h}$, and $\lambda_{h}>\hat{\kappa}_{h+1}$, since $\lambda_{h}>\kappa_{h}>\hat{\kappa}_{\bar{h}}=\hat{\kappa}_{h+1}$. So

(4) $\hat{\kappa}_{h+1}=\hat{\kappa}_{i}$.

This leads to the contradiction

$$
\kappa_{h}>\hat{\kappa}_{\bar{h}}=\hat{\kappa}_{i} \geq \lambda_{\bar{h}}>\kappa_{h}
$$

Now that we know that $\xi<\mathcal{T} i$, the second part of the claim follows quite easily: We know that $\hat{\kappa}_{i}=i_{\xi, i}^{\mathcal{T}}\left(\hat{\kappa}_{\xi}\right)$. So we have to show that $\hat{\kappa}_{\xi}<\operatorname{crit}\left(i_{\xi, i}^{\mathcal{T}}\right)$. But otherwise, $\hat{\kappa}_{i} \geq \lambda_{i}^{\mathcal{T}}$, which contradicts the fact that $\xi=\widehat{\mathcal{T}}(i+1)$, so that, in particular, $\hat{\kappa}_{i}<\lambda_{\xi}^{\mathcal{T}}$.

Proof of $(b)$ This is vacuously true if $\xi=i$. So let $\xi<i$. Assume the claim to be false. Let $h+1$ be a counterexample, and let $\zeta=\mathcal{T}(h+1)$. Then

$$
\xi \leq \mathcal{T} \zeta<\mathcal{T} h+1 \leq \mathcal{T} i, \quad \zeta=\hat{T}(h+1)
$$

By (a), $\hat{\kappa}_{h}=\hat{\kappa}_{\zeta}$, and $\hat{\kappa}_{i}=\hat{\kappa}_{\xi}$.

Now, $\hat{\kappa}_{i}=\hat{\kappa}_{\xi} \leq \hat{\kappa}_{\zeta}=\hat{\kappa}_{h}$ (since $\xi \leq \mathcal{T} \zeta$ ). On the other hand, $\hat{\kappa}_{i} \geq \hat{\kappa}_{\zeta}=\hat{\kappa}_{h}$ (since $\zeta \leq \mathcal{T} i$ ). So $\hat{\kappa}_{\xi}=\hat{\kappa}_{\zeta}=\hat{\kappa}_{h}=\hat{\kappa}_{i}$. But this leads to the contradiction

$$
\hat{\kappa}_{i} \geq \hat{\kappa}_{h+1}=i_{\zeta, h+1}^{\mathcal{T}}\left(\hat{\kappa}_{\zeta}\right)=i_{\zeta, h+1}^{\mathcal{T}}(\underbrace{\hat{\kappa}_{h}}_{=\kappa_{h}^{\mathcal{T}}})>\hat{\kappa}_{h}=\hat{\kappa}_{i} .
$$

The construction in the proof of the main lemma also relies on two commutativity properties of coarse ultrapowers. We state them as separate lemmas. The first one is an extension of [5, Lemma 2.7], albeit for a coarse structural setting. The corresponding fine structural versions of our lemmas don't hold true, and this is why our main result talks about coarse (normal) iterability. 
Lemma 3.2 Let $M, M^{\prime}$ be premice. Let $\bar{\sigma}: M \longrightarrow_{F} M^{\prime}$, where $F=E_{\text {top }}^{M}$. Let $H$ be a (possibly long) extender on $M$ and $M^{\prime}$ s.t., letting $\pi: M \longrightarrow_{H} N, \pi^{\prime}: M^{\prime} \longrightarrow_{H}$ $N^{\prime}, \pi \subseteq \pi^{\prime}$. Let $G=E_{\text {top }}^{N}$ and $\tilde{\lambda}=\operatorname{lh}(H) \leq \kappa=\operatorname{crit}(G)$. Let $\sigma: N \longrightarrow_{G} \tilde{N}$. Then $\tilde{N}=N^{\prime}$ and $\pi^{\prime} \bar{\sigma}=\sigma \pi$.

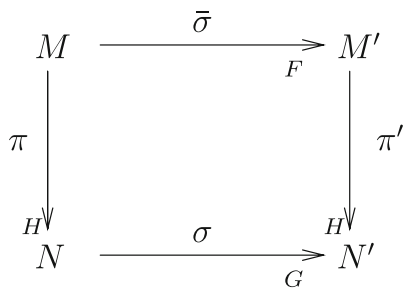

Proof Let $\bar{\kappa}=\operatorname{crit}(F)$. Let us first argue that $G \circ \pi$ and $\pi \circ F$ agree on $\mathcal{P}(\bar{\kappa}) \cap M$. Let $X \in \mathcal{P}(\bar{\kappa}) \cap M$. Then

$$
F(X)=Y \Longleftrightarrow G(\pi(X))=\pi(Y)
$$

by the elementarity of $\pi$. In other words, $G \circ \pi(X)=\pi \circ G(X)$.

We now define a $\Sigma_{0}$-preserving map $\tau: \tilde{N} \longrightarrow N^{\prime}$ and show that it is surjective. We deduce how it must be defined. To this end, let $a \in \tilde{N}$ be given. It is of the form $a=\sigma(f)(\vec{\alpha})$, where $f \in \kappa^{m} N \cap N$ and $\vec{\alpha}<v(G)(\vec{\alpha}$ are generators of $G)$. And $f$ is of the form $f=\pi(g)(\vec{\gamma})$, where $g \in \tilde{\kappa}^{n} M \cap M, \vec{\gamma}<\tilde{\lambda}(\operatorname{dom}(H) \subseteq \mathcal{P}(\tilde{\kappa}))$. Let $\psi$ be a $\Sigma_{0}$ formula. We get:

$$
\begin{aligned}
& \tilde{N} \models \psi[\sigma(f)(\vec{\alpha})] \\
& \Longleftrightarrow \quad \prec \vec{\alpha} \succ \in G(\{\prec \vec{\beta} \succ<\kappa|N| \psi[f(\vec{\beta})]\}) \\
& \Longleftrightarrow \quad \prec \vec{\alpha} \succ \in G(\{\prec \vec{\beta} \succ<\kappa \mid N \models \psi[\pi(g)(\vec{\gamma})(\vec{\beta})]\}) \\
& \underset{\vec{\gamma}<\tilde{\lambda} \leq \kappa}{\Longleftrightarrow} \quad \prec \vec{\alpha}, \vec{\gamma} \succ \in G(\{\prec \vec{\beta}, \vec{\delta} \succ<\kappa \mid N \models \psi[\pi(g)(\vec{\delta})(\vec{\beta})]\}) \\
& \Longleftrightarrow \quad \prec \vec{\alpha}, \vec{\gamma} \succ \in G(\pi(\{\prec \vec{\beta}, \vec{\delta} \succ<\bar{\kappa} \mid M \models \psi[g(\vec{\delta})(\vec{\beta})]\}))
\end{aligned}
$$

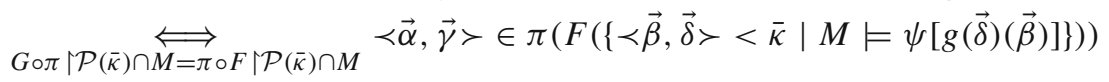

$$
\begin{aligned}
& \Longleftrightarrow \quad \prec \vec{\alpha}, \vec{\gamma} \succ \in \pi^{\prime}(\bar{\sigma}(\{\prec \vec{\beta}, \vec{\delta} \succ<\bar{\kappa} \mid M \models \psi[g(\vec{\delta})(\vec{\beta})]\})) \\
& \Longleftrightarrow \quad N^{\prime} \models \psi[\underbrace{\pi^{\prime}(\bar{\sigma}(g))(\gamma)(\vec{\alpha})}_{:=\tau(\sigma(f)(\vec{\alpha}))}]
\end{aligned}
$$

Remember that $\vec{\alpha}<\operatorname{lh}(G)$ and $\vec{\gamma}<\tilde{\lambda}$. To see that $\tau$ is onto, let an arbitrary element $b$ of $N^{\prime}$ be given. It is of the form $b=\pi^{\prime}(f)(\vec{\gamma})$, where $f \in \tilde{\kappa}^{m} M^{\prime} \cap M^{\prime}$ and $\vec{\gamma}<\tilde{\lambda}$. And $f$ is of the form $f=\bar{\sigma}(g)(\vec{\alpha})$, where $g \in \bar{\kappa}^{n} M \cap M, \vec{\alpha}<\lambda(F)$. So

$$
\begin{aligned}
b & =\pi^{\prime}(\bar{\sigma}(g))\left(\pi^{\prime}(\vec{\alpha})\right)(\vec{\gamma}) \\
& =\pi^{\prime}(\bar{\sigma}(g))(\underbrace{\pi(\vec{\alpha})}_{<\operatorname{lh}(G)})(\underbrace{\vec{\gamma}}_{<\tilde{\lambda}}) .
\end{aligned}
$$


This is clearly in the range of $\tau$; cf. its definition. So $\tau=\operatorname{id}\left\lceil\tilde{N}\right.$, hence $\tilde{N}=N^{\prime}$, and also:

$$
\sigma(\pi(g)(\vec{\gamma}))(\vec{\alpha})=\pi^{\prime}(\vec{\sigma}(g))(\vec{\gamma})(\vec{\alpha}),
$$

which readily implies that $\sigma \pi=\pi^{\prime} \bar{\sigma}$.

Lemma 3.3 Let $M, M^{\prime}$ be premice. Let $\bar{\sigma}: M \longrightarrow{ }_{F} M^{\prime}$, where $F=E_{\text {top }}^{M}$ and $\bar{\kappa}=\operatorname{crit}(F)$. Let $H$ be a long extender of length $\tilde{\lambda}$ on $M, M^{\prime}$ s.t., letting $\pi: M \longrightarrow_{H}$ $N, \pi^{\prime}: M^{\prime} \longrightarrow H N^{\prime}, \pi \subseteq \pi^{\prime}$. Let $G=E_{\text {top }}^{N}$ and suppose that $\bar{\kappa}<\operatorname{crit}(\pi)(=$ $\left.\operatorname{crit}\left(\pi^{\prime}\right)\right)$ and $\tilde{\lambda} \leq \operatorname{lh}(G)($ so $\kappa:=\operatorname{crit}(G)=\bar{\kappa})$.

Let $\sigma: M \longrightarrow G \tilde{N}$. Then $\tilde{N}=N^{\prime}$ and $\pi^{\prime} \bar{\sigma}=\sigma$.

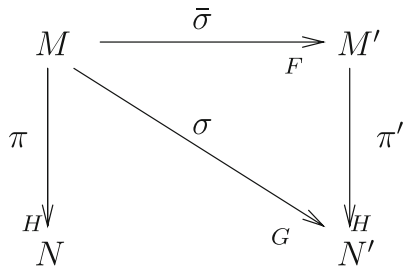

Proof We proceed as in the previous lemma. We again have that $G \circ \pi$ and $\pi \circ F$ agree on $\mathcal{P}(\bar{\kappa}) \cap M$. So we define $\tau: \tilde{N} \longrightarrow \Sigma_{0} N^{\prime}$ and show that $\tau$ is surjective.

So let $a \in \tilde{N}$ be given. It's of the form $a=\sigma(f)(\vec{\alpha})$, where $f \in \kappa^{\kappa^{m}} M \cap M$ and $\vec{\alpha}<\lambda(G)$. Let $\psi$ be a $\Sigma_{0}$ formula. We get:

$$
\begin{aligned}
& \tilde{N} \models \psi[\sigma(f)(\vec{\alpha})] \\
& \Longleftrightarrow \quad \prec \vec{\alpha} \succ \in G(\underbrace{\{\prec \vec{\beta} \succ<\kappa \mid M \models \psi[f(\vec{\beta})]\}}_{:=x \subseteq \kappa<\operatorname{crit}(\pi)})
\end{aligned}
$$

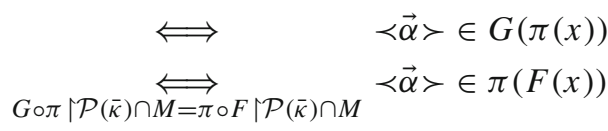

$$
\begin{aligned}
& \Longleftrightarrow \quad \prec \vec{\alpha} \succ \in \pi^{\prime}(\bar{\sigma}(x)) \\
& \Longleftrightarrow \quad N^{\prime} \vDash \psi \psi[\underbrace{\pi^{\prime} \bar{\sigma}(f)(\vec{\alpha})}_{:=\tau(\sigma(f)(\vec{\alpha}))}] .
\end{aligned}
$$

Note that $\vec{\alpha}<\lambda(G) . \tau$ is designed to be $\Sigma_{0}$ preserving. To see that it is onto, let $b$ be an arbitrary element of $N^{\prime}$. It has the form $b=\pi^{\prime}(f)(\vec{\alpha})$, for some $f \in{ }^{\tilde{\kappa}} M^{\prime} \cap M^{\prime}$ and $\vec{\alpha}<\tilde{\lambda} \leq \operatorname{lh}(G)$. And $f$ can be rendered as $f=\bar{\sigma}(g)(\vec{\gamma})$, where $g \in \bar{\kappa}^{m} M \cap M$ and $\vec{\gamma}<\lambda(F)$. So

$$
\begin{aligned}
b & =\pi^{\prime}(\bar{\sigma}(g))\left(\pi^{\prime}(\vec{\gamma})\right)(\vec{\alpha}) \\
& =\pi^{\prime}(\bar{\sigma}(g))(\underbrace{\pi(\vec{\gamma})}_{<\lambda(G)})(\underbrace{\vec{\alpha}}_{<\tilde{\lambda}}),
\end{aligned}
$$


which is in the range of $\tau$. So $\tau=\operatorname{id}\left\lceil\tilde{N}, \tilde{N}=N^{\prime}\right.$ and

$$
\pi^{\prime} \bar{\sigma}(f)(\vec{\alpha})=\sigma(f)(\vec{\alpha})
$$

which obviously implies that $\pi^{\prime} \bar{\sigma}=\sigma$.

\section{The copying construction}

We are now ready to prove the main result of this article.

The iterability notions used in the statement of the following theorem are defined in the Introduction.

Main Theorem 4.1 Let $M$ be an active premouse. If $M$ is separately $\alpha+1$-iterable, then $M$ is coarsely normally $\alpha+1$-iterable.

Proof Fix a normal iteration strategy $\Sigma$ for the $\alpha$ th top iterate of $M$. We shall describe an iteration strategy $\Sigma^{\prime}$ for coarse normal iterations of $M$.

The idea is that the iteration strategy for $M$ will be as follows: If an iteration tree on $M$ of limit length is according to the strategy we are about to describe, then construct a "copy" of the iteration tree onto the $\alpha$ th iterate of $M$, apply $\Sigma$ to that iteration, and pull back the branch it gives. The iteration tree on the $\alpha$ th top iterate of $M$ is not allowed to use the top extender, since $M$ is only separately iterable. The idea is that we shift all the applications of the top extender in the original tree to the beginning of the copied tree. It is here that the two commutativity Lemmas of the previous section come in.

We shall now describe the copying process.

Let $\mathcal{T}$ be an iteration tree on $\mathcal{M}$. Write $M_{i}$ for $\mathcal{M}_{i}^{\mathcal{T}}$.

Let $N_{0}^{i}=\left\langle\left|N_{0}^{i}\right|, \in, F_{0}^{i}\right\rangle$ be the $i$ th top iterate of $M, \kappa_{0}^{i}=\operatorname{crit}\left(F_{0}^{i}\right), \lambda_{0}^{i}=\lambda\left(F_{0}^{i}\right)$, and let $\sigma_{i, j}^{0}: N_{0}^{i} \longrightarrow N_{0}^{j}$ be the iteration embedding $(i \leq j \leq \alpha)$. Let $N_{0}=\left(N_{0}^{\alpha}\right)^{\text {passive }}$.

We shall construct a kind of copy of $\mathcal{T}$ onto $N_{0}$. The resulting iteration tree on $N_{0}$ will be called $\mathcal{U}=c(\mathcal{T})$. The models in that tree will be referred to as $N_{i}=\mathcal{M}_{i}^{\mathcal{U}}$. Along with $\mathcal{U}$, we shall define a function $\varphi: \ln (\mathcal{T}) \longrightarrow \ln (\mathcal{T})$ and premice $N_{i}^{\gamma}$, for $\gamma \leq \alpha$ and $i+1<\operatorname{lh}(\mathcal{U})$. The construction proceeds by induction.

Set:

At stage $i$ we shall define $\nu_{i}^{\mathcal{U}}, \varphi(i), \mathcal{M}_{i}^{\mathcal{U}}, \mathcal{U}(i)$, and the premice $N_{i}^{\gamma}$, for $\gamma \leq \alpha$.

1. $N_{i}=\mathcal{M}_{i}^{\mathcal{U}}$.

2. $N_{i}^{\gamma}=i_{0, i}^{\mathcal{U}}\left(N_{0}^{\gamma}\right)$.

More precisely, $\left|N_{i}^{\gamma}\right|=i_{0, i}^{\mathcal{U}}\left(\left|N_{0}^{\gamma}\right|\right)$, and $N_{i}^{\gamma}=\left\langle\left|N_{i}^{\gamma}\right|, \in, F_{i}^{\gamma}\right\rangle$, where $F_{i}^{\gamma}=$ $\bigcup_{x \in\left|N_{0}^{\gamma}\right|} i_{0, i}^{\mathcal{U}}\left(F_{0}^{\gamma} \cap x\right)$.

3. $\kappa_{i}^{j}=\operatorname{crit}\left(F_{i}^{j}\right), \lambda_{i}^{j}=\lambda\left(F_{i}^{j}\right), v_{i}^{j}=v\left(F_{i}^{j}\right)$.

We prove inductively at every stage $i$ :

(1) $M_{i}=N_{i}^{\varphi(i)}$.

(2) If there are no truncations in $[0, i]_{\mathcal{T}}$, then, setting $\xi=\hat{T}(i+1)$, the following hold true: 
(a) $\varphi(i)=\varphi(\xi)$ and $\xi \leq \mathcal{T} i$.

(b) $N_{i}^{\varphi(\xi)+1}=\operatorname{Ult}\left(N_{\xi}^{\varphi(\xi)}, F_{i}^{\varphi(i)}\right)$. Furthermore, setting

$$
\pi_{\xi, i}^{\prime}: N_{\xi}^{\varphi(\xi)} \longrightarrow F_{i}^{\varphi(i)} N_{i}^{\varphi(\xi)+1}
$$

and letting $\sigma_{\varphi(\xi), \varphi(\xi)+1}^{\xi}$ be the embedding from $N_{\xi}^{\varphi(\xi)}$ into its coarse ultrapower by $F_{\xi}^{\varphi(\xi)}$, we have:

$$
\sigma_{\varphi(\xi), \varphi(\xi)+1}^{\xi}: N_{\xi}^{\varphi(\xi)} \longrightarrow{ }_{F_{\xi}^{\varphi(\xi)}} N_{\xi}^{\varphi(\xi)+1}
$$

and $\pi_{\xi, i}^{\prime}=i_{\xi, i}^{\mathcal{U}} \sigma_{\varphi(\xi), \varphi(\xi)+1}^{\xi}$.

(c) $\left\langle N_{i}^{\gamma} \mid \gamma \in[\varphi(i)+1, \alpha]\right\rangle$ are the models in the top iteration of $N_{i}^{\varphi(i)+1}$. Denoting the iteration embeddings by $\sigma_{\mu, v}^{i}: N_{i}^{\mu} \longrightarrow N_{i}^{v}$, we have:

$$
\sigma_{\mu, \nu}^{i} i_{\xi, i}^{\mathcal{U}}=i_{\xi, i}^{\mathcal{U}} \sigma_{\mu, v}^{\xi}
$$

The following diagram illustrates the situation:
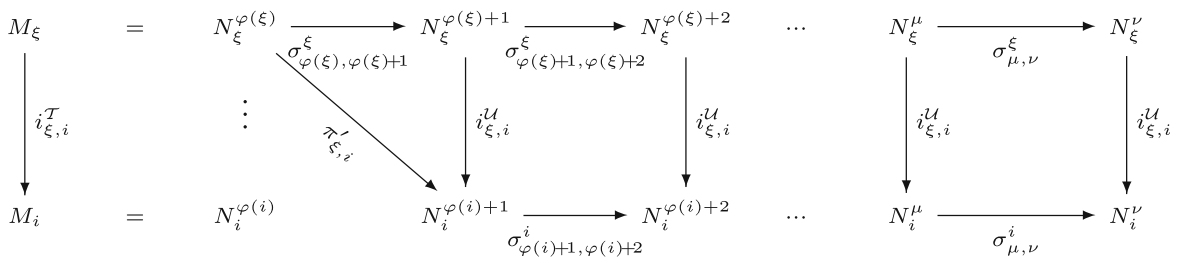

(3) (a) Let $j<\mathcal{T} i$. Then $i_{j, i}^{\mathcal{T}}=i_{j, i}^{\mathcal{U}} \sigma_{\varphi(j), \varphi(i)}^{j}$.

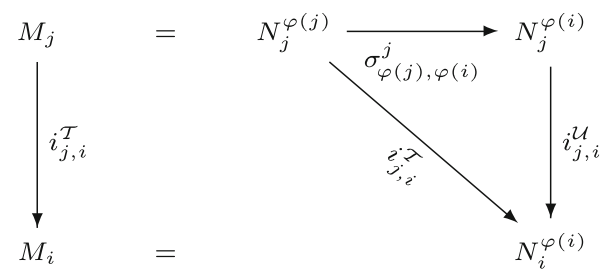

(b) Let $j<\mathcal{U} i$. Then $i_{j, i}^{\mathcal{U}} \sigma_{\gamma, \delta}^{j}=\sigma_{\gamma, \delta}^{i} i_{j, i}^{\mathcal{U}}$, for $\varphi(i)+1 \leq \gamma \leq \delta$. If $\hat{T}(i+1)=i$, then this holds for $\varphi(i) \leq \gamma \leq \delta$ as well.
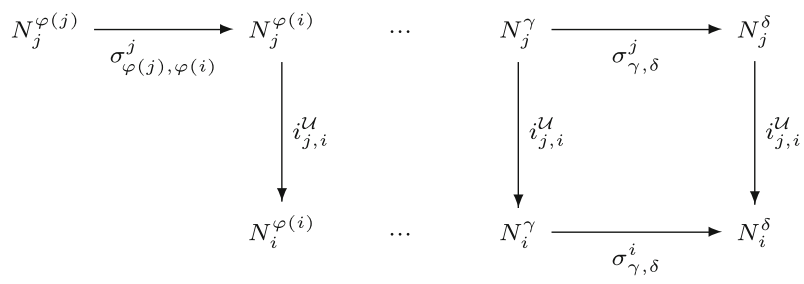
A remark on condition (3)(a) is in order here: If $\gamma:=\varphi(j)=\varphi(i)$, then we take $\sigma_{\gamma, \gamma}^{j}$ to be the identity. But if $\varphi(j)<\varphi(i)$, then it is not at all clear that the embed$\operatorname{ding} \sigma_{\varphi(j), \varphi(i)}^{j}$ is defined. But in this case, $\hat{T}(j+1)=j$, as we shall show presently: Let $k^{\prime}$ be minimal s.t. $j \leq \mathcal{T} k^{\prime} \leq \mathcal{T} i$ and $\varphi\left(k^{\prime}\right)>\varphi(j)$. Then $k^{\prime}$ will be of the form $k+1, v_{h}=\hat{v}_{h}$ and $\varphi(k+1)=\varphi(k)+1=\varphi(j)+1$ (and hence $T(k+1)=\hat{T}(k+1))$, as will follow from our construction. So $j<\mathcal{T} k+1 \leq \mathcal{T} i$. We show that $j=\hat{T}(k+1)$. Otherwise, $j<\mathcal{T} \xi:=T(k+1)$ and $\kappa_{k}=\hat{\kappa}_{k} \geq \lambda_{j}$. We know by Lemma 3.1 that $\hat{\kappa}_{k}=\hat{\kappa}_{\xi}$, and that $\xi \leq \mathcal{T} k$. So we have: $j<\mathcal{T} \xi \leq_{\mathcal{T}} k$. But since $\hat{T}(j+1)<j, \hat{\kappa}_{j}<\sup _{\gamma<j} \lambda_{\gamma} \leq \operatorname{crit}\left(i_{j, \xi}^{\mathcal{T}}\right)$. But this means that $\hat{\kappa}_{j}=\hat{\kappa}_{\xi}<\lambda_{j}$, a contradiction. So this shows that $\hat{T}(k+1)=j$. But this implies, again by Lemma 3.1, that $\hat{\kappa}_{j}=\hat{\kappa}_{k}$, and hence that $\hat{T}(j+1)=\hat{T}(k+1)=j$, which is what we wanted to show. So the existence of the embedding $\sigma_{\varphi(j), \varphi(i)}^{j}$ in (3)(a) is not problematic after all, since it follows from the items (2)(b) and (2)(c) at stage $j$.

Turning to the construction, it is no surprise that we set $\varphi(0)=0$. If $v_{0}^{\mathcal{T}} \in \mathcal{M}_{0}^{\mathcal{T}}$, then $v_{0}^{\mathcal{U}}=v_{0}^{\mathcal{T}}$. Otherwise, $v_{0}^{\mathcal{U}}$ is undefined; $\mathcal{U}$ is a padded tree.

If the stages $j<i$ have been constructed already, we shall describe how to define the $i$ th stage.

First, consider the case that $i$ is a successor.

Then we let $v_{i}^{\mathcal{U}}$ be undefined iff $E_{i}^{\mathcal{T}}=E_{\text {top }}^{\mathcal{M}_{i}^{\mathcal{T}}}$ and there were no truncations in $[0, i)_{\mathcal{T}}$ (so that $E_{i}^{\mathcal{T}}$ is the "image of the top extender of $\mathcal{M}_{0}^{\mathcal{T}}$ under the embedding $\left.i_{0, i}^{\mathcal{T}}\right)$ ". Otherwise, we let $v_{i}^{\mathcal{U}}=v_{i}^{\mathcal{T}}$.

In order to define $\mathcal{U}(i), \mathcal{M}_{i}^{\mathcal{U}}$ and $\varphi(i)$, we distinguish two cases:

If $\nu_{i-1}^{\mathcal{U}}$ is defined, then we $\operatorname{set} \mathcal{U}(i)=\mathcal{T}(i)$ (inductively, we will see that $\mathcal{M}_{\mathcal{U}(i)}^{\mathcal{U}}=$ $\mathcal{M}_{\xi}^{\mathcal{U}}$ where $\xi$ is minimal s.t. either $\xi=i-1$, or $\xi<i-1$ and $\kappa_{i-1}^{\mathcal{U}}<\lambda_{\xi}^{\mathcal{U}}$. In general, it does not have to be the case that $\xi=\mathcal{U}(i)$, though. So $\mathcal{U}$ is not, verbatim, a padded normal iteration tree. But it is not far from being such a tree; it is a liberally padded normal iteration tree, as we shall call it).

This determines $\mathcal{M}_{i}^{\mathcal{U}}$. In this case, we also set $\varphi(i)=\varphi(\mathcal{T}(i))$.

If $v_{i-1}^{\mathcal{U}}$ is undefined, we $\operatorname{set} \mathcal{U}(i)=i-1$, and let $\varphi(i)=\varphi(\mathcal{T}(i))+1$.

In the case that $i$ is a limit, we wish to apply $\Sigma$ to the part of the tree $\mathcal{U}$ constructed so far (note that the copying procedure depends on the iteration strategy $\Sigma$ that we fixed in advance).

This tree will always be a liberally padded normal iteration tree; see the next section, where it is also shown that the iteration strategy for $N_{0}$ works for such trees.

So if this tree is according to $\Sigma$, this iteration strategy can be applied, yielding a cofinal well founded branch $b$ through it.

Let $\mathcal{L}$ be the set of $i$ such that $v_{i}^{\mathcal{U}}$ is undefined. Let

$$
\mathcal{E}=\{j \in \mathcal{L} \mid \forall \xi<j \exists \zeta \in[\xi, j) \quad \zeta \notin \mathcal{L}\}
$$

and set $\mathcal{E}+1=\{i+1 \mid i \in \mathcal{E}\}$. We shall define a putative branch $b^{\prime}$ from $b$ by cases:

( $\star$ If $\mathcal{E}+1 \cap b$ is bounded, say by $\xi$, then we let $b^{\prime}$ be the $<\mathcal{T}$-closure of $b \backslash(\xi+1)$. If not, we let $b^{\prime}$ be the $<\mathcal{T}$-closure of $\mathcal{E}+1 \cap b$. 
If $b^{\prime}$ is a cofinal wellfounded branch of the part of $\mathcal{T}$ constructed so far, then we extend $\mathcal{T}$ by $b^{\prime}$ and set $\varphi(i)=\sup _{j<\mathcal{T} i} \varphi(j)$. Otherwise the construction breaks down.

We shall show that this is a successful coarse normal iteration strategy. First, we are going to verify our inductive hypotheses (1)-(3).

For $i=0$, nothing has to be shown.

Now suppose (1)-(3) hold for every $j<i$. We show they hold for $i$ as well.

Main Case 1: $i$ is a successor ordinal.

Proof of (1) Let $\xi=T(i)$. If $\nu_{i-1}^{\mathcal{U}}$ is defined, then $E_{i-1}^{\mathcal{U}}=E_{i-1}^{\mathcal{T}}$ and $\xi=\mathcal{U}(i)$. By induction hypothesis, $M_{\xi}=N_{\xi}^{\varphi(\xi)}$. Now, $v_{\xi}^{\varphi(\xi)}$ is a regular cardinal in $N_{\xi}$ (since it is the image of $\nu_{0}^{\varphi(\xi)}$ under the elementary embedding $i_{0, \xi}^{\mathcal{U}}$ ). So $i_{\xi, i}^{\mathcal{T}}=i_{\xi, i}^{\mathcal{U}}\left\lceil N_{\xi}^{\varphi(\xi)}\right.$, and thus

$$
N_{i}^{\varphi(\xi)}=\operatorname{Ult}\left(N_{\xi}^{\varphi(\xi)}, E_{i-1}^{\mathcal{T}}\right)=\operatorname{Ult}\left(M_{\xi}, E_{i-1}^{\mathcal{T}}\right)=M_{i}
$$

But $\varphi(\xi)=\varphi(i)$, so we are done.

Now suppose $v_{i-1}^{\mathcal{U}}$ is undefined. So the top extender was applied in $\mathcal{T}$ in the $(i-1)$ st step, $\xi=T(i)=\hat{T}(i), \varphi(i)=\varphi(i-1)+1$, and there was no truncation in $[0, i-1]_{\mathcal{T}}$. But now, (2) holds at stage $i-1$. By (a), we know that $\varphi(\xi)=\varphi(i-1)$, and by (b), $N_{i-1}^{\varphi(\xi)+1}=\operatorname{Ult}\left(N_{\xi}^{\varphi(\xi)}, F_{i-1}^{\varphi(i-1)}\right)$. But by (1) at stage $i-1$, we know that $M_{i-1}=$ $N_{i-1}^{\varphi(i-1)}$, so in particular, $E_{i-1}^{\mathcal{T}}=F_{i-1}^{\varphi(i-1)}$. Putting these together, we see:

$$
M_{i}=\operatorname{Ult}\left(M_{\xi}, E_{i-1}^{\mathcal{T}}\right)=\operatorname{Ult}\left(N_{\xi}^{\varphi(\xi)}, F_{i-1}^{\varphi(i-1)}\right)=N_{i-1}^{\varphi(\xi)+1}
$$

But $N_{i-1}=N_{i}$ and $\varphi(i)=\varphi(i-1)+1=\varphi(\xi)+1$, so $M_{i}=N_{i}^{\varphi(i)}$, as was to be shown.

Now we turn to the verification of (2). In fact, the proof given works for limit $i$ as well.

Proof of (2) So let $\xi=\hat{T}(i+1)$. To verify (2)(a), note that $\xi \leq \mathcal{T} i$ by Lemma 3.1, part (a). Part (b) of that lemma shows that $\varphi(i)=\varphi(\xi)$, because the only stages at which $\varphi(j)<\varphi\left(j^{\prime}\right)$, where $j=T\left(j^{\prime}\right)$ are those with $v_{j^{\prime}}^{\mathcal{T}}=\hat{v}_{j^{\prime}}^{\mathcal{T}}$. Such stages don't exist in $(\xi, i]$, so $\varphi(\xi) \geq \varphi(i)$. But it is easy to verify that $\varphi$ is weakly monotonous along branches. So $\varphi(\xi)=\varphi(i)$.

It also follows that

$$
\xi=\hat{T}(\xi+1) .
$$

This is because we know from Lemma 3.1 that $\hat{\kappa}_{\xi}=\hat{\kappa}_{i}$. Hence, $\hat{T}(\xi+1)=\hat{T}(i)=\xi$. Case 1: $\xi<i$

Then, because $\xi=T(\xi+1)$, we know that $\left\langle N_{\xi}^{\gamma} \mid \gamma \in[\varphi(\xi), \alpha]\right\rangle$ is the top iteration of $N_{\xi}^{\varphi(\xi)}$. This is what our inductive hypothesis (2) at stage $\xi$ gives us. Set: 
$\tilde{\lambda}:=\sup \left\{\lambda_{j} \mid j+1 \in(\xi, i]_{\mathcal{T}}\right\} \leq \hat{\lambda}_{i}$, and $\tilde{\kappa}=\min \left\{\delta \mid \tilde{\lambda} \leq i_{\xi, i}^{\mathcal{U}}(\delta)\right\}\left(\tilde{\lambda} \leq \hat{\lambda}_{i}\right.$ because the former is a limit cardinal in $M_{i}$ and the latter is the largest cardinal in $M_{i} . \tilde{\kappa}$ exists because $i_{\xi, i}^{\mathcal{T}}$ is cofinal).

We have the following situation: $\sigma_{\varphi(i), \varphi(i)+1}^{\xi}: N_{\xi}^{\varphi(i)} \longrightarrow{ }_{F_{\xi}^{\varphi(i)}} N_{\xi}^{\varphi(i)+1}$, and $N_{i}^{\varphi(i)}$ is the ultrapower of $N_{\xi}^{\varphi(i)}$ by the long extender $i_{\xi, i}^{\mathcal{U}} \mid \tilde{\kappa}, \hat{\kappa}_{i}^{\varphi(i)}=\operatorname{crit}\left(F_{i}^{\varphi(i)}\right)=\hat{\kappa}_{i}=\hat{\kappa}_{\xi}=$ $\operatorname{crit}\left(F_{\xi}^{\varphi(\xi)}\right)=\hat{\kappa}_{\xi}^{\varphi(\xi)}<\operatorname{crit}\left(i_{\xi, i}^{\mathcal{U}}\right)$ and $\tilde{\lambda} \leq \hat{\lambda}_{i}=\operatorname{lh}\left(F_{i}^{\varphi(i)}\right)=\hat{\kappa}_{i}^{\varphi(i)}$. So Lemma 3.3 can be applied and gives (2)(b).

The corresponding argument, but applying Lemma 3.2, gives (2)(c). To see this, argue by induction on $\gamma \geq \varphi(i)+1$. Assume that we inductively know that $\left\langle N_{i}^{\delta} \mid \delta \in[\varphi(i)+1, \gamma]\right\rangle$ are the models in an initial segment of the top iteration of $N_{i}^{\varphi(i)+1}$. Call the iteration embeddings $\sigma_{\mu, \nu}^{i}(\varphi(i)+1 \leq \mu \leq \nu \leq \gamma)$. Then we have the situation $\sigma_{\gamma, \gamma+1}^{\xi}: N_{\xi}^{\gamma} \longrightarrow{ }_{F_{\xi}^{\gamma}} N_{\xi}^{\gamma+1}$, and $N_{i}^{\gamma}, N_{i}^{\gamma+1}$ are the ultrapowers of $N_{\xi}^{\gamma}, N_{\xi}^{\gamma+1}$, resp., by the long extender $i_{\xi, i}^{\mathcal{U}} \uparrow \mathcal{P}(\tilde{\kappa})$. We have just seen in the previous paragraph that $\tilde{\lambda} \leq \hat{\lambda}_{i}^{\varphi(i)}$. But $\hat{\lambda}_{i}^{\varphi(i)}=\hat{\kappa}_{i}^{\varphi(i)+1} \leq \sigma_{\varphi(i)+1, \gamma}^{i}\left(\hat{\kappa}_{i}^{\varphi(i)+1}\right)=\hat{\kappa}_{i}^{\gamma}$ (note that, inductively, the embedding $\sigma_{\varphi(i)+1, \gamma}^{i}$ exists). This is all that's required in order to be able to apply Lemma 3.2. It gives that $N_{i}^{\gamma+1}=\operatorname{Ult}\left(N_{i}^{\gamma}, F_{i}^{\gamma}\right)$ and that, denoting the ultrapower embedding by $\sigma_{\gamma, \gamma+1}^{i}, \sigma_{\gamma, \gamma+1}^{i} i_{\xi, i}^{\mathcal{U}}=i_{\xi, i}^{\mathcal{U}} \sigma_{\gamma, \gamma+1}^{\xi}$. Defining $\sigma_{\mu, \gamma+1}^{i}=\sigma_{\gamma, \gamma+1}^{i} \sigma_{\mu, \gamma}^{i}$ and $\sigma_{\gamma+1, \gamma+1}^{i}=\mathrm{id}$, it's obvious that these embeddings commute nicely: $\sigma_{\mu, v}^{i} v_{\xi, i}^{\mathcal{U}}=i_{\xi, i}^{\mathcal{U}} \sigma_{\mu, \nu}^{\xi}$, for $\varphi(i)+1 \leq \mu \leq v \leq \gamma+1$. We will make use of this commutativity when dealing with the limit case.

So let $\gamma$ be a limit ordinal. Let

$$
\left\langle\tilde{N}, \sigma_{\mu, \lambda}^{i}\right\rangle=\operatorname{dir} \lim \left(\left\langle N_{i}^{\mu} \mid \varphi(i)+1 \leq \mu<\gamma\right\rangle,\left\langle\sigma_{\mu, \nu}^{i} \mid \varphi(i)+1 \leq \mu \leq v<\gamma\right\rangle\right) .
$$

We derive how to define an embedding $\tau: \tilde{N} \longrightarrow \Sigma_{0} N_{i}^{\gamma}$. Let $\psi$ be a $\Sigma_{0}$ formula which holds in $\tilde{N}$ of $a=\sigma_{\mu, \lambda}^{i}(\bar{a})$. Letting $\bar{a}=i_{\xi, i}^{\mathcal{U}}(f)(\vec{\alpha})$, where $f \in \tilde{\kappa}^{m} N_{\xi}^{\mu} \cap N_{\xi}^{\mu}$ and $\vec{\alpha}<\tilde{\lambda}$, we get:

$$
\begin{aligned}
\tilde{N} \models \psi[a] & \Longleftrightarrow N_{i}^{\mu} \models \psi[\bar{a}] \\
& \Longleftrightarrow \prec \vec{\alpha} \succ \in i_{\xi, i}^{\mathcal{U}}\left(\left\{\prec \vec{\beta} \succ<\tilde{\kappa} \mid N_{\xi}^{\mu} \models \psi[f(\vec{\beta})]\right\}\right) \\
& \Longleftrightarrow \prec \vec{\alpha} \succ \in i_{\xi, i}^{\mathcal{U}}\left(\sigma_{\mu, \lambda}^{\xi}\left(\left\{\prec \vec{\beta} \succ<\tilde{\kappa} \mid N_{\xi}^{\mu} \models \psi[f(\vec{\beta})]\right\}\right) \cap \tilde{\kappa}\right) ;
\end{aligned}
$$

the last equivalence is because $\tilde{\kappa} \leq \hat{\lambda}_{\xi}=\hat{\kappa}_{\xi}^{\varphi(i)+1} \leq \hat{\kappa}_{\xi}^{\mu}$ (we already know that $\tilde{\lambda} \leq \hat{\lambda}_{i}^{\varphi(i)}$. So $i_{\xi, i}^{\mathcal{U}}\left(\hat{\lambda}_{\xi}\right)=\hat{\lambda}_{i} \geq \tilde{\lambda}$. Now $\tilde{\kappa}=\min \left\{\delta \mid \tilde{\lambda} \leq i_{\xi, i}^{\mathcal{T}}(\delta)\right\}$, i.e., $\tilde{\kappa} \leq \hat{\lambda}_{\xi}$.). Hence, we get:

$$
\begin{aligned}
\tilde{N} \models \psi[a] & \Longleftrightarrow \prec \vec{\alpha} \succ \in i_{\xi, i}^{\mathcal{U}}\left(\left\{\langle\vec{\beta} \succ<\tilde{\kappa}| N_{\xi}^{\lambda} \models \psi\left[\sigma_{\mu, \lambda}^{\xi}(f)(\vec{\beta})\right]\right\}\right) \\
& \Longleftrightarrow N_{i}^{\lambda} \models \psi[\underbrace{\mathcal{U}}_{:=\tau(a)} \underbrace{}_{\xi, i}\left(\sigma_{\mu, \lambda}^{\xi}(f)(\vec{\alpha})\right] .
\end{aligned}
$$


That $\tau$ is well defined follows from the commutativity properties mentioned above. To see that it is onto, note that every element $b$ of $N_{i}^{\lambda}$ is of the form $i_{\xi, i}^{\mathcal{U}}(f)(\vec{\alpha})$, for some $f^{\prime} \in \tilde{\kappa}^{m} N_{\xi}^{\lambda} \cap N_{\xi}^{\lambda}$ and $\vec{\alpha}<\tilde{\lambda}$. Now $f^{\prime}=\sigma_{\mu, \lambda}^{\xi}(f)$, for some $\mu \in[\varphi(i)+1, \lambda), f \in N_{\xi}^{\mu}$. Then, setting $b^{\prime}=\sigma_{\mu, \lambda}^{i}\left(i_{\xi, i}^{\mathcal{U}}\left(f^{\prime}\right)(\vec{\alpha})\right)$, we have: $b=\tau\left(b^{\prime}\right)$, and this shows that $N_{i}^{\lambda}=\tilde{N}$. Moreover, since $\tau$ is the identity, this means, for $a$ as above:

$$
\begin{aligned}
a & =\sigma_{\mu, \lambda}^{i}\left(i_{\xi, i}^{\mathcal{U}}(f)(\vec{\alpha})\right) \\
& =\sigma_{\mu, \lambda}^{i}\left(i_{\xi, i}^{\mathcal{U}}(f)\right)(\vec{\alpha}) \\
& =i_{\xi, i}^{\mathcal{U}}\left(\sigma_{\mu, \lambda}^{\xi}(f)(\vec{\alpha}),\right.
\end{aligned}
$$

which clearly implies that $\sigma_{\mu, \lambda}^{i} i_{\xi, i}^{\mathcal{U}}=i_{\xi, i}^{\mathcal{U}} \sigma_{\mu, \lambda}^{\xi}$, as wished.

Case 2: $\xi=i$.

Then $\hat{\kappa}_{i} \geq \lambda_{j}$, for all $j<i$. So letting $\tilde{\lambda}:=\sup \left\{\lambda_{j} \mid j+1 \leq \mathcal{T} i\right\} \leq \hat{\lambda}_{i}$, and $\tilde{\kappa}=\min \left\{\delta \mid \tilde{\lambda} \leq i_{\xi, i}^{\mathcal{T}}(\delta)\right\} \leq \hat{\lambda}_{0}=\hat{\kappa}_{0}^{1}$. So we can apply Lemma 3.2 to the long extender $i_{0, i}^{\mathcal{U}} \mid \mathcal{P}(\tilde{\kappa})$, whose generators are bounded by $\tilde{\lambda} \leq \hat{\kappa}_{i}^{\varphi(i)}$. Of course, $\left\langle N_{0}^{\gamma} \mid \gamma \in[\varphi(i), \alpha]\right\rangle$ are the models in the top iteration of $N_{0}^{\varphi(i)}$, by definition. The argument is just like in case 1 . We get that $\left\langle N_{\gamma}^{i} \mid \gamma \in[\varphi(i), \alpha]\right\rangle$ are the models in the top iteration of $N_{\varphi(i)}^{i}$, which shows (2)(b) and (2)(c) at once. Note that (2)(a) is trivial in this case.

Proof of (3) We omit the proof of (3)(b) because as a matter of fact, it is implicit in the proof of (2)(b) in case 1 . Replace $\xi$ there by an arbitrary $j<\mathcal{U} i$. The proof goes thru.

To prove (3)(a), we again distinguish two subcases. Let $\xi=T(i)$.

Case 1: $v_{i-1}^{\mathcal{U}}$ is defined.

Then $\xi=\mathcal{U}(i), \varphi(\xi)=\varphi(i), \sigma_{\varphi(\xi), \varphi(i)}^{\xi}$ is the identity, and $i_{\xi, i}^{\mathcal{T}}=i_{\xi, i}^{\mathcal{U}}\left\lceil N_{\xi}^{\varphi(\xi)}\right.$. So we have trivially:

$$
i_{\xi, i}^{\mathcal{T}}=i_{\xi, i}^{\mathcal{U}} \sigma_{\varphi(\xi), \varphi(i)}^{\xi}
$$

This shows one instance of (3)(a). Now let $j<\mathcal{T} i$. If $j=\xi$, we have just shown what there is to show. Otherwise, $j<\mathcal{T} \xi<\mathcal{T} i$. We know that (3)(a) holds at stage $\xi$, giving us:

$$
i_{j, \xi}^{\mathcal{T}}=i_{j, \xi}^{\mathcal{U}} \sigma_{\varphi(j), \varphi(\xi)}^{j}
$$

Hence,

$$
\begin{aligned}
i_{j, i}^{\mathcal{T}} & =i_{\xi, i}^{\mathcal{T}} i_{j, \xi}^{\mathcal{T}} \\
& =i_{\xi, i}^{\mathcal{U}} i_{j, \xi}^{\mathcal{U}} \sigma_{\varphi(j), \varphi(\xi)}^{j} \\
& =i_{j, i}^{\mathcal{U}} \sigma_{\varphi(j), \varphi(\xi)}^{j},
\end{aligned}
$$


which is what is claimed in (3)(a).

Case 2: $v_{i-1}^{\mathcal{U}}$ is undefined.

So in this case, $\varphi(i)=\varphi(i-1)+1, \mathcal{U}(i)=i-1, v_{i}=\hat{v}_{i}$ and $\hat{T}(i)=\mathcal{T}(i)=\xi$. We first put (2)(a) and (b) at stage $i-1$ to use. By (2)(a), $\varphi(\xi)=\varphi(i-1)$ and $\xi \leq \mathcal{T} i-1$.

Noting that $i_{\xi, i}^{\mathcal{T}}: N_{\xi}^{\varphi(\xi)} \longrightarrow{ }_{F_{i-1}^{\varphi(i-1)}} N_{i}^{\varphi(i)}=N_{i-1}^{\varphi(\xi)+1}$, i.e., $i_{\xi, i}^{\mathcal{T}}=\pi_{\xi, i-1}^{\prime}$, (2)(b) says that

$$
i_{\xi, i}^{\mathcal{T}}=i_{\xi, i-1}^{\mathcal{U}} \sigma_{\varphi(\xi), \varphi(\xi)+1}^{\xi}
$$

But $i_{\xi, i-1}^{\mathcal{U}}=i_{\xi, i}^{\mathcal{U}}$ and $\varphi(\xi)+1=\varphi(i)$, so this says:

$$
i_{\xi, i}^{\mathcal{T}}=i_{\xi, i}^{\mathcal{U}} \sigma_{\varphi(\xi), \varphi(i)}^{\xi},
$$

which is one instance of (3)(a). To prove the full property (3)(a), let $j<\mathcal{T} i$. If $j=\xi$, we're done. So let $j<\mathcal{T} \xi$. Applying (3)(a) at stage $\xi$ gives:

$$
i_{j, \xi}^{\mathcal{T}}=i_{j, \xi}^{\mathcal{U}} \sigma_{\varphi(j), \varphi(\xi)}^{j}
$$

Finally, $\xi=\hat{T}(\xi+1)$ and hence $\hat{\kappa}_{\xi}=\hat{\kappa}_{\xi}^{\varphi(\xi)} \geq \tilde{\lambda}=\sup _{l<\xi \lambda_{l}}$. So Lemma 3.2 can be applied to give

$$
i_{j, \xi}^{\mathcal{U}} \sigma_{\varphi(\xi), \varphi(\xi)+1}^{j}=\sigma_{\varphi(\xi), \varphi(\xi)+1}^{\xi} i_{j, \xi}^{\mathcal{U}}
$$

Putting the last three displayed equations together, we get:

$$
\begin{aligned}
i_{j, i}^{\mathcal{T}} & =i_{\xi, i}^{\mathcal{T}} i_{j, \xi}^{\mathcal{T}} \\
& =i_{\xi, i}^{\mathcal{U}} \sigma_{\varphi(\xi), \varphi(i)}^{\xi} i_{j, \xi}^{\mathcal{U}} \sigma_{\varphi(j), \varphi(\xi)}^{j} \\
& =i_{\xi, i}^{\mathcal{U}} i_{j, \xi}^{\mathcal{U}} \sigma_{\varphi(\xi), \varphi(\xi)+1}^{j} \sigma_{\varphi(j), \varphi(\xi)}^{j} \\
& =i_{j, i}^{\mathcal{U}} \sigma_{\varphi(j), \varphi(i)}^{j},
\end{aligned}
$$

as wished. We used that $\varphi(i)=\varphi(\xi)+1$ here.

Main Case 2: $i$ is a limit ordinal.

In this case, it suffices to prove (1) and (3), as the proof of (2) given in the successor case works in the limit case just as well.

The proof of (1) is where property (3) will come in. We will define a $\Sigma_{0}$ preserving embedding $\tau: \mathcal{M}_{b^{\prime}}^{\mathcal{T}} \longrightarrow N_{i}^{\varphi(i)}$. Remember that $\varphi(i)=\sup _{j \in b} \varphi(j)$. So let $a \in \mathcal{M}_{b^{\prime}}^{\mathcal{T}}$. We will describe where $a$ should be mapped. Being an element of the direct limit, $a$ has a preimage $\bar{a}=\left(i_{j, b^{\prime}}^{\mathcal{T}}\right)^{-1}(a)$ in some $M_{j}$, where $j \in b^{\prime}$. Set:

$$
\tau(a)=i_{j, \lambda}^{\mathcal{U}} \sigma_{\varphi(j), \varphi(\lambda)}^{j}(\bar{a}) .
$$


Note that this makes sense since at least one of the following hold: a) $\varphi(j)=\varphi(\lambda)$, or b) $j=\hat{T}(j+1)$. For if a) fails, then b) holds, because $j$ has $\mathcal{T}$-successor (cf. the remark after the statement of property (3)(a)). In any case, $\sigma_{\varphi(j), \varphi(\lambda)}^{j}$ is defined, and all of this is indeed a definition of a function since the embeddings involved commute as described in (3)(b). A routine verification shows that $\tau$ is onto, and hence that (1) is satisfied at stage $i$. That $\tau$ is surjective indeed means that $\tau$ is the identity, and hence, it follows from the definition of $\tau$ that

$$
i_{j, \lambda}^{\mathcal{T}}(\bar{a})=a=\tau(a)=i_{j, \lambda}^{\mathcal{U}} \sigma_{\varphi(j), \varphi(\lambda)}^{j}(\bar{a}),
$$

which is precisely what is demanded in (3)(a). (3)(b) is again implicit in the proof of (2)(c) in the successor case.

This finishes the construction.

Let us look back and see what we have achieved so far: We fixed an iteration strategy $\Sigma$ for the passive version of the top iterate $N$ of $M$ of stage $\alpha$. Then we described a partial function $c$ on coarse normal iteration trees on $M$. The $\alpha$-iteration strategy $\Sigma^{\prime}$ for $M$, which is claimed to exist by the theorem, is described as follows: Given a coarse normal iteration tree $\mathcal{T}$ of limit length on $M$, form the copy $\mathcal{U}=c(\mathcal{T})$, which is a padded tree on $N$. If this is defined, then it is a liberally padded normal coarse iteration tree on $N$ (this notion is defined in the next section). If $\mathcal{U}$ is according to $\Sigma$, and is of limit length, then let $b=\Sigma(\mathcal{U})$. Let $b^{\prime}$ be defined from $b$ as described in $(\star)$. If this is a cofinal well founded branch of $\mathcal{T}$, then set $\Sigma^{\prime}(\mathcal{T})=b^{\prime}$. Otherwise, $\Sigma^{\prime}(\mathcal{T})$ is undefined.

In order to see that this is a successful coarse normal $\alpha$-iteration strategy for $M$, the following has to be shown: If $\mathcal{T}$ is a coarse normal iteration tree on $M$ which is according to $\Sigma^{\prime}$ and has length $<\alpha$, then

1. If $\mathcal{T}$ has length $\theta+1, v$ indexes an extender in $\mathcal{M}_{\theta}^{\mathcal{T}}$ with critical point $\kappa$, and $v>v_{i}^{\mathcal{T}}$, for any $i<\theta$, then, letting $\xi$ be least s.t. $\kappa<\lambda_{\xi}^{\mathcal{T}}$ and $\zeta$ be maximal s.t. $F=E_{v}^{\mathcal{M}_{\theta}^{\mathcal{T}}}$ is total on $\mathcal{M}^{*}=\mathcal{M}_{\xi}^{\mathcal{T}} \| \zeta$, then $\operatorname{Ult}\left(\mathcal{M}^{*}, F\right)$ is well-founded.

2. If $\mathcal{T}$ has limit length, then $c(\mathcal{T})$ exists and is according to $\Sigma$, in a sense which is made precise in the next section.

3. Further, if $b=\Sigma(c(\mathcal{T}))$, and $b^{\prime}$ is defined from $b$ as in $(\star)$, then $b^{\prime}$ is a cofinal well-founded branch of $\mathcal{T}$.

We shall postpone these points to the end of the next section. Modulo these, the proof of the Main Theorem is complete.

Corollary 4.2 If $M$ is coarsely separately $\alpha$-iterable, for every $\alpha$, and if the sequence of the corresponding iteration strategies $\left\langle\Sigma_{\alpha} \mid \alpha<\infty\right\rangle$ is definable, where $\Sigma_{\alpha}$ is an $\alpha+1$-iteration strategy of the coarse top iterate of stage $\alpha$ of $M$, then $M$ is coarsely iterable.

Proof The proof of the Main Theorem shows how to convert the $\alpha+1$-iteration strategy $\Sigma_{\alpha}$ of the $\alpha$ th top iterate of $M$ into a coarse $\alpha$-iteration strategy $\Sigma_{\alpha}^{\prime}$ of $M$, in a uniform way. So the sequence $\left\langle\Sigma_{\alpha}^{\prime} \mid \alpha<\infty\right\rangle$ is definable. We can now define a coarse normal $\infty$-iteration strategy $\Sigma$ for $M$, as follows: Given a coarse normal iteration tree 
on $M$, we let $\alpha(\mathcal{T})$ be the least ordinal $\alpha$ with the property that $b=\Sigma_{\alpha}^{\prime}(\mathcal{T})$ is defined and for unboundedly many $\alpha^{\prime}, \Sigma_{\alpha^{\prime}}^{\prime}(\mathcal{T})=b$, if such an $\alpha$ exists. If $\alpha(\mathcal{T})$ exists, we let $\Sigma(\mathcal{T})=\Sigma_{\alpha(\mathcal{T})}^{\prime}(\mathcal{T})$. It is straightforward to check that $\Sigma$ is a successful coarse normal iteration strategy.

\section{The relationship between $\mathcal{T}$ and its "copy"}

In the following, we will try to capture the kind of iteration tree that our "copy" $c(\mathcal{T})$ of $\mathcal{T}$ is.

Definition 5.1 A liberally padded strongly normal iteration tree $\mathcal{T}$ is a padded iteration tree of some length $\theta$ with a set $\mathcal{L}$ of "lazy points" (this is just the set of $i$ such that $v_{i}^{\mathcal{T}}$ is undefined), in which the indices of extenders applied are strictly increasing, and the rules for choosing predecessors are as follows:

A If $i$ is not lazy, then let $\xi=\min \left(\{i\} \cup\left\{j<i \mid j \notin \mathcal{L} \wedge \kappa_{i}^{\mathcal{T}}<\lambda_{j}^{\mathcal{T}}\right\}\right)$. We demand that $\mathcal{M}_{\mathcal{T}(i+1)}^{\mathcal{T}}=\mathcal{M}_{\xi}^{\mathcal{T}}$.

B If $i$ is lazy, then $\mathcal{T}(i+1)=i$.

There is a subtle difference between this notion of a padded tree and that given in [2]. There, if $\alpha \in \mathcal{L}$, then $\alpha<\mathcal{T} \beta$ iff $\beta=\alpha+1$ or $\alpha+1<_{\mathcal{T}} \beta$, whereas in the case of liberal padding it is possible that the tree branches at $\alpha \in \mathcal{L}$.

This is only a mild difference, though, and it is quite easy to see that a normal iteration strategy $\Sigma$ for a premouse $\mathcal{M}$ can be extended to an iteration strategy which works for liberally padded iteration trees on $\mathcal{M}$. For given an iteration tree $\mathcal{T}$ on $\mathcal{M}$ which is liberally padded, we can form a "condensed" version $\mathcal{T}$ ' of $\mathcal{T}$, which can be viewed as indexed by equivalence classes of the equivalence relation on $\ln (\mathcal{T})$ which identifies ordinals indexing the same models. Enumerating those equivalence classes in such a way that predecessors in the tree order come first, the resulting tree is a coarse normal iteration tree. So $\Sigma$ can be applied to that tree, giving a cofinal well-founded branch $b$ through $\mathcal{T}^{\prime}$. The interesting case now is that $\mathcal{T}^{\prime}$ has limit length. In that case, one can convert $b$ into a cofinal branch through the original tree $\mathcal{T}$ as follows: Let $c$ be the the set of $\mathcal{T}$-predecessors of least members of equivalence classes of $b$, and then let $c^{\prime}$ be the branch generated by $c$. It is cofinal, and $\mathcal{M}_{c}^{\mathcal{T}}=\mathcal{M}_{b}^{\mathcal{T}^{\prime}}$.

This justifies the application of $\Sigma$ to $\mathcal{U}$ in the proof of Theorem 4.1, since it is clear that $\mathcal{U}$ is liberally padded. So at this point, the construction of $c(\mathcal{T})$ is really done.

We shall need some more observations in order to prove the remaining three points mentioned at the end of the proof of Theorem 4.1.

For this section, fix a coarse normal tree $\mathcal{T}$ and $\mathcal{U}=c(\mathcal{T})$. Set

$$
\mathcal{L}=\left\{i<\theta \mid v_{i}^{\mathcal{T}}=\hat{v}_{i}^{\mathcal{T}} \text { and there are no drops in }[0, i)_{\mathcal{T}}\right\}
$$

This happens to be the set of lazy points of $\mathcal{U}$. Let $\mathfrak{I}$ be the set of maximal intervals consisting of points $i \in \mathcal{L}$. In order to gain a better understanding of $\mathcal{U}$, let's introduce

$$
\mathfrak{S}=\{\theta \cap(I \cup\{\operatorname{lub} I\}) \mid I \in \mathfrak{I}\} .
$$


Here, we use lub $A$ for the strict supremum of $A$, i.e., the least ordinal strictly greater than all members of $A$, if $A$ is nonempty, 0 otherwise. It follows that $\mathfrak{S}$ is pairwise disjoint. Hence we can define, for $i<\theta$,

$$
I_{i}:=\left\{\begin{array}{cl}
I & \text { if } I \text { is the unique } I \in \mathfrak{S} \text { s.t. } i \in I, \\
\{i\} & \text { if no such } I \text { exists. }
\end{array}\right.
$$

Set $i \simeq j$ iff $I_{i}=I_{j}$. Hence, $\mathcal{M}_{i}^{\mathcal{U}}=\mathcal{M}_{j}^{\mathcal{U}}$ iff $i \simeq j$, and $I_{i}$ is the equivalence class of $i$ with respect to $\simeq$.

This gives an alternative definition of the set $\mathcal{E}$ introduced in the course of the proof of Theorem 4.1, to wit:

$$
\mathcal{E}=\{\min I \mid I \in \mathfrak{S}\}
$$

So $\mathcal{E}$ consists of lazy points that begin a new interval of lazy points. We shall also set:

$$
\mathcal{E}+1=\{i+1 \mid i \in \mathcal{E}\}
$$

Suppose for the rest of this section that $\mathcal{T}$ is a coarse normal simple iteration tree on $\mathcal{M}$ which is in accordance with the strategy $\Sigma^{\prime}$ described in the previous section. For the reader's convenience we restate the choice of the branches on the $\mathcal{T}$-side: If $i$ is a limit ordinal, then, letting $b=[0, i)_{\mathcal{U}}=\Sigma\left(\mathcal{U}\lceil i)\right.$, we have that $[0, i)_{\mathcal{T}}=b^{\prime}$, where $b^{\prime}$ is defined as follows:

If $\mathcal{E}+1 \cap[0, i)_{\mathcal{U}}$ is bounded, say by $\xi$, then $b^{\prime}$ is the closure of $b \backslash(\xi+1)$ under $<\mathcal{T}$.

Otherwise, $b^{\prime}$ is the closure of $\mathcal{E}+1 \cap b$ under $<\mathcal{T}$.

Lemma 5.2 Let $i+1<\theta=\operatorname{lh}(\mathcal{U})$.

(a) If $i \notin \mathcal{L}$, then $\mathcal{U}(i+1)=\mathcal{T}(i+1)$.

(b) If $i \in \mathcal{L}$ and $i \notin \mathcal{E}$, then $\mathcal{T}(i+1)=i=\mathcal{U}(i+1)$.

So, if $j<\theta$ is a successor ordinal which is not a member of $\mathcal{E}+1$, then $\mathcal{T}(j)=$ $\mathcal{U}(j)$.

Proof Claim (a) is obvious.

For (b), assume the contrary. Let $i$ be the minimal counterexample.

If $i$ is a successor, we know that $i-i \in \mathcal{L}$, so $\hat{\kappa}_{i-1}=\hat{\kappa}_{\mathcal{T}}(i)$, by Lemma 3.1. Hence $\kappa_{i}^{\mathcal{T}}=\hat{\kappa}_{i}=\hat{\lambda}_{i-1}=\lambda_{i-1}^{\mathcal{T}}$. So $\mathcal{T}(i+1)=i=\mathcal{U}(i+1)$.

Now let $i$ be a limit. Since $i \in \mathcal{L}$ but $i \notin \mathcal{E}, \xi:=\min I_{i}<i$. Obviously then, every $\alpha \in I_{i} \cap i$ is a $<\mathcal{U}$-predecessor of $i$. By the translation of branches from $\mathcal{U}$ to $\mathcal{T}$, every $\alpha \in I_{i} \cap i$ which is greater than $\xi$, is also a $\mathcal{T}$-predecessor of $i$.

Now we show that $\kappa_{i} \geq \lambda_{\alpha}$ for every $\alpha \in\left(i \cap I_{i}\right) \backslash\{\xi\}$. Let such an $\alpha$ be given. Then $\alpha<\mathcal{T} \alpha+1<\mathcal{T} i$, as we now know. So $\hat{\kappa}_{\alpha+1}=i_{\alpha, \alpha+1}^{\mathcal{T}}\left(\hat{\kappa}_{\alpha}\right)=\hat{\lambda}_{\alpha}$, by Lemma 3.1. So $\hat{\kappa}_{i} \geq i_{\alpha+1, i}^{\mathcal{T}}\left(\hat{\kappa}_{\alpha+1}\right) \geq \hat{\lambda}_{\alpha}$. But $\kappa_{i}^{\mathcal{T}}=\hat{\kappa}_{i}$ and $\lambda_{\alpha}^{\mathcal{T}}=\hat{\lambda}_{\alpha}$, so we are done.

In the following, we shall adopt the common convention of writing $[0, i)_{\mathcal{T}}$ for the set of all $j$ such that $j<\mathcal{T} i$, and analogously for $\mathcal{U}$. 


\section{Lemma $5.3<\mathcal{T} \subseteq<\mathcal{U}$.}

Proof We show by induction on $i<\operatorname{lh}(\mathcal{T})$ that $[0, i)_{\mathcal{T}} \subseteq[0, i)_{\mathcal{U}}$.

This is obvious for $i=0$.

Suppose we have shown the claim for all $j \leq i$. We prove it for $i+1$. To do this, we first check that $\mathcal{T}(i+1)<\mathcal{U} i+1$ :

Case 1: $i \in \mathcal{L}$.

Then $\mathcal{T}(i+1)=\widehat{\mathcal{T}}(i+1) \leq \mathcal{T} i$. By induction hypothesis, it follows that $\mathcal{T}(i+1) \leq \mathcal{U} i$. Since $i \in \mathcal{L}, i<\mathcal{U} i+1$, so $\mathcal{T}(i+1)<\mathcal{U} i+1$, as claimed.

\section{Case 2: $i \notin \mathcal{L}$.}

$$
\text { Then } \mathcal{T}(i+1)=\mathcal{U}(i+1)<\mathcal{U} i+1 \text {. }
$$

Now $[0, i+1)_{\mathcal{T}}=\{\mathcal{T}(i+1)\} \cup[0, \mathcal{T}(i+1))_{\mathcal{T}}$. By induction hypothesis, $[0, \mathcal{T}(i+$ 1) $)_{\mathcal{T}} \subseteq[0, \mathcal{T}(i+1))_{\mathcal{U}}$. But since $\mathcal{T}(i+1)<_{\mathcal{U}} i+1,[0, \mathcal{T}(i+1))_{\mathcal{T}} \subseteq[0, i+1)_{\mathcal{U}}$, and, finally, $\{\mathcal{T}(i+1)\} \cup[0, \mathcal{T}(i+1)) \mathcal{U} \subseteq[0, i+1) \mathcal{U}$.

Now let $i$ be a limit ordinal. Let $b=[0, i)_{\mathcal{U}}$ and $b^{\prime}=[0, i)_{\mathcal{T}}$. Since $\mathcal{U}=c(\mathcal{T})$, we know that $b^{\prime}$ is the $<\mathcal{T}$-closure of some subset of $b$. But then, the inductive hypothesis yields that $b^{\prime} \subseteq b$, so we are done. do:

We now need some kind of a converse to the fact that $<\mathcal{T} \subseteq<\mathcal{U}$. The following will

Lemma 5.4 If $i \leq \mathcal{U} j$ and $(\mathcal{E}+1) \cap(i, j]_{\mathcal{U}}=\emptyset$, then $i \leq \mathcal{T} j$.

Proof This follows from Lemma 5.2, by induction on $j$. Or, put differently, deny, and let $j$ be the least counterexample. Then $i<\mathcal{U} j$.

From the abovementioned Lemma it follows that $j$ cannot be a successor ordinal. For if it were, then by Lemma 5.2, $\mathcal{T}(j)=\mathcal{U}(j)$, and since $(\mathcal{E}+1) \cap(i, \mathcal{U}(j)]=\emptyset$, it follows by minimality of $j$ that $i \leq \mathcal{T} \mathcal{U}(j)$. But $\mathcal{U}(j)=\mathcal{T}(j)<\mathcal{T} j$, so $i<\mathcal{T} j$, contradicting our assumption of the contrary.

So $j$ is a limit. Let $b=[0, j)_{\mathcal{U}}$. Since $\mathcal{E}+1 \cap b$ is bounded by $i+1$ in $j$, it follows that $b^{\prime}=[0, j)_{\mathcal{T}}$ is the closure of $b \backslash i+1$ under $<\mathcal{T}$. So, in particular, $i<\mathcal{T} j$ after all: Letting $h$ be the immediate $<\mathcal{U}$-successor of $i$ in $b, i$ is a successor not in $\mathcal{E}+1$, so $i=\mathcal{U}(h)=\mathcal{T}(h) \in b^{\prime}$.

Lemma 5.5 Let $i_{0} \in \mathcal{E}$, and $i_{0}+1 \leq \mathcal{U} i_{1} \in \mathcal{E}$. Further, assume that $\mathcal{E}+1 \cap\left(i_{0}+\right.$ $\left.1, i_{1}\right]=\emptyset$. Then $\mathcal{T}\left(i_{1}+1\right)>i_{0}$.

Proof By Lemma 5.4, it follows that $i_{0}+1 \leq \mathcal{T} i_{1}$. So

$$
\hat{\kappa}_{i_{0}+1} \leq \hat{\kappa}_{i_{1}} .
$$

Since $i_{0} \in \mathcal{E}, \lambda_{i_{0}}=\hat{\lambda}_{i_{0}}=\hat{\kappa}_{i_{0}+1}$ (we used Lemma 3.1 here). And $i_{1} \in \mathcal{E}$, so $\kappa_{i_{1}}=\hat{\kappa}_{i_{1}}$. Hence, $\kappa_{i_{1}} \geq \lambda_{i_{0}}$, or, in other words, $\mathcal{T}\left(i_{1}+1\right)>i_{0}$.

Lemma 5.6 Let $i_{0}+1, i_{1}+1 \in \mathcal{E}+1$ and $i_{0}+1 \leq \mathcal{U} i_{1}+1$. Then $i_{0}+1 \leq \mathcal{T} i_{1}+1$. 
Proof Deny. Fix $i_{0}$ and let $i_{1}$ be the least counterexample.

Then $\left(i_{0}+1, i_{1}+1\right]_{\mathcal{U}} \cap \mathcal{E}+1 \neq \emptyset$. Because otherwise, by Lemma 5.5, it follows that $\mathcal{T}\left(i_{1}+1\right) \geq i_{0}+1$. But by Lemma 5.3, $\mathcal{T}\left(i_{1}+1\right)<\mathcal{U} i_{1}+1$. So, both $i_{0}+1$ and $T\left(i_{1}+1\right)$ are $<_{\mathcal{U}}$-predecessors of $i_{1}+1$, and $i_{0}+1 \leq \mathcal{T}\left(i_{1}+1\right)$. This implies that

$$
i_{0}+1 \leq_{\mathcal{U}} \mathcal{T}\left(i_{1}+1\right)
$$

But $\mathcal{E}+1 \cap\left(i_{0}+1, \mathcal{T}\left(i_{1}+1\right)\right] \mathcal{U}=\emptyset$, so by Lemma $5.4, i_{0}+1 \leq \mathcal{T}\left(i_{1}+1\right)$. Of course, $\mathcal{T}\left(i_{1}+1\right)<\mathcal{T} i_{1}+1$, so we get that $i_{0}+1 \leq \mathcal{T} i_{1}+1$, after all.

Moreover, $\mathcal{E}+1 \cap\left(i_{0}+1, i_{1}\right]_{\mathcal{U}}$ has no maximal element. For suppose $\mu+1$ were maximal in that set. Note that since $i_{1} \in \mathcal{E}, i_{1} \notin \mathcal{E}+1$. So $\mu+1<i_{1}$, and

$$
\mathcal{E}+1 \cap\left(\mu+1, i_{1}\right] \mathcal{U}=\emptyset .
$$

By the argument in the previous paragraph, replacing $i_{0}$ with $\mu$, it follows that

$$
\mu+1<\mathcal{T} i_{1}+1
$$

And since $i_{1}$ is the least counterexample for the claim of the lemma, we have that

$$
i_{0}+1 \leq \mathcal{T} \mu+1
$$

which shows that $i_{0}+1<\mathcal{T} i_{1}+1$ after all.

So let $\sigma=\sup \left(\mathcal{E}+1 \cap\left(i_{0}+1, i_{1}\right] \mathcal{U}\right)$. Let $c=\left[0, i_{1}\right] \mathcal{U} \cap \sigma$. Since $\sigma$ is a limit of $c, \sigma<\mathcal{U} i_{1}$, and hence, $c=[0, \sigma) \mathcal{U}$. It follows that, letting $c^{\prime}$ be the $<\mathcal{T}$-hull of $c \cap(\mathcal{E}+1), c^{\prime}=[0, \sigma)_{\mathcal{T}}$. So,

$$
i_{0}+1<\mathcal{T} \sigma<\mathcal{U} i_{1}+1
$$

But since $\left(\sigma, i_{1}\right] \cap \mathcal{E}+1=\emptyset$, we know by Lemma 5.4 that $\sigma \leq \mathcal{T} i_{1}$. So,

$$
i_{0}+1<\mathcal{T} i_{1}
$$

Now, repeating the argument from Lemma 5.5, we show that

$$
\mathcal{T}\left(i_{1}+1\right) \geq \sigma
$$

For otherwise we could pick $h+1 \in \mathcal{E}+1 \cap\left(i_{0}+1, \sigma\right) \mathcal{U}$ so that $\kappa_{i_{1}}<\lambda_{h}$. But $\kappa_{i_{1}}=\hat{\kappa}_{i_{1}}$ and, since $h+1 \in \mathcal{E}+1, \lambda_{h}=\hat{\kappa}_{h+1}$, which, since $h+1<\mathcal{T} i_{1}$, is at most $\hat{\kappa}_{i_{1}}$. So $\kappa_{i_{1}} \geq \lambda_{h}$, a contradiction.

So, both $\sigma$ and $\mathcal{T}\left(i_{1}+1\right)$ are $<\mathcal{U}$-predecessors of $i_{1}+1$, and $\sigma \leq \mathcal{T}\left(i_{1}+1\right)$, which shows that $\sigma \leq_{\mathcal{U}} \mathcal{T}\left(i_{1}+1\right)$. But $\left(\sigma, \mathcal{T}\left(i_{1}+1\right)\right] \cap \mathcal{E}+1=\emptyset$, so

$$
i_{0}+1<_{\mathcal{T}} \sigma \leq \mathcal{T} \mathcal{T}\left(i_{1}+1\right)<\mathcal{T} i_{1}+1
$$

which contains the claim to the failure of which $i_{1}$ was chosen to be a witness. 
Now we are ready to verify that the strategy $\Sigma^{\prime}$ described in the proof of the Main Theorem is a successful coarse normal $\alpha$-iteration strategy for $\mathcal{M}$. So we have to verify the points mentioned at the end of that proof; we reproduce them here, for the reader's convenience. If $\mathcal{T}$ is a coarse normal iteration tree on $M$ which is according to $\Sigma^{\prime}$ and has length $<\alpha$, then we have to show:

1. If $\mathcal{T}$ has length $\theta+1, v$ indexes an extender in $\mathcal{M}_{\theta}^{\mathcal{T}}$ with critical point $\kappa$, and $v>v_{i}^{\mathcal{T}}$, for any $i<\theta$, then, letting $\xi$ be least s.t. $\kappa<\lambda_{\xi}^{\mathcal{T}}$ and $\zeta$ be maximal s.t. $F=E_{v}^{\mathcal{M}_{\theta}^{\mathcal{T}}}$ is total on $\mathcal{M}^{*}=\mathcal{M}_{\xi}^{\mathcal{T}} \| \zeta$, then $\operatorname{Ult}\left(\mathcal{M}^{*}, F\right)$ is well-founded.

2. If $\mathcal{T}$ has limit length, then $c(\mathcal{T})$ exists and is according to $\Sigma$.

3. Further, if $b=\Sigma(c(\mathcal{T}))$, and $b^{\prime}$ is defined from $b$ as in $(\star)$, then $b^{\prime}$ is a cofinal well-founded branch of $\mathcal{T}$. (The definition of $b^{\prime}$ will be repeated in the proof below.)

The first of these points is clear, since the construction of $c(\mathcal{T})$ shows that $c\left(\mathcal{T}^{\prime}\right)$ exists, where $\mathcal{T}^{\prime}$ is the one-point extension described in that point.

The second point is clear as well, since for every limit $\lambda<\operatorname{lh}(\mathcal{T})$, the branch $[0, \lambda)_{\mathcal{T}}$ was picked by $\Sigma^{\prime}$, which means that $[0, \lambda)_{c(\mathcal{T} \mid \lambda)}$ was picked by $\Sigma$.

For the last point: We have to show that $b^{\prime}$ is a cofinal well-founded branch of $\mathcal{T}$, where $b^{\prime}$ is defined by cases, as follows.

The first case is that $[0, i) \mathcal{U} \cap(\mathcal{E}+1)$ is bounded in $i$. In that case, if $\xi$ is such a bound, then $b^{\prime}$ is the closure of $b \backslash(\xi+1)$ under $<\mathcal{T}$. For $\alpha, \beta \in b$ such that $\alpha<\mathcal{U} \beta$, it follows from Lemma 5.4 that $\alpha<\mathcal{T} \beta$. So $b^{\prime}$ is a cofinal branch of $\mathcal{T}$.

In the second case, $[0, i) \mathcal{U} \cap(\mathcal{E}+1)$ is cofinal in $i$. In that case, $b^{\prime}$ is the $<\mathcal{T}$-closure of $b \cap(\mathcal{E}+1)$. By Lemma 5.6, if $\alpha<\beta$ both are elements of $b \cap(\mathcal{E}+1)$, then $\alpha<\mathcal{T} \beta$. So in that case too, $b^{\prime}$ is a cofinal branch.

That $b^{\prime}$ is well-founded follows from the construction of $\mathcal{T}$ and $\mathcal{U}$ : The universe of the limit $\mathcal{M}_{b^{\prime}}^{\mathcal{T}}$ is the same as that of $N_{i}^{\varphi(i)}$, in the terminology of the proof of the main theorem.

This completes the proof of Theorem 4.1.

Open Access This article is distributed under the terms of the Creative Commons Attribution Noncommercial License which permits any noncommercial use, distribution, and reproduction in any medium, provided the original author(s) and source are credited.

\section{References}

1. Jensen, R.: A New Fine Structure for Higher Core Models. Handwritten Notes, Berlin (1997)

2. Mitchell, W.J., Steel, J.R.: Fine Structure and Iteration Trees. Lecture Notes in Logic vol. 3. Springer, Berlin (1994)

3. Steel, J.R.: The Core Model Iterability Problem. Lecture Notes in Logic vol. 8. Springer, Berlin (1996)

4. Steel, J.R.: An outline of inner model theory. In: Foreman, M., Kanamori, A., Magidor, M. Handbook of Set Theory, Springer, Berlin (2009)

5. Steel, J.R., Welch, P.D.: $\Sigma_{3}^{1}$ absoluteness and the second uniform indiscernible. Israel J. Math. 104, 157-190 (1998)

6. Schindler, R., Zeman, M.: Fine structure. In: Foreman, M., Kanamori, A., Magidor, M. Handbook of Set Theory, Springer, Berlin (2009)

7. Zeman, M.: Inner Models and Large Cardinals. De Gruyter, Berlin (2002) 